Technological University Dublin ARROW@TU Dublin

2007-01-01

\title{
Microstructures and Viscoelastic Properties of Anisotropic Magnetorheological Elastomers
}

\author{
Lin Chen \\ Technological University Dublin, lin.chen@tudublin.ie
}

Follow this and additional works at: https://arrow.tudublin.ie/cerart

Part of the Mechanics of Materials Commons, and the Polymer and Organic Materials Commons

\section{Recommended Citation}

Chen, L., Gong, X.L., \& Li, W.H. (2007) Microstructures and Viscoelastic Properties of Anisotropic MR Elastomers. Smart Materials \& Structures, 16(6): 2645-2650, 2007. doi:10.21427/D7W322

This Article is brought to you for free and open access by the Centre for Elastomer Research at ARROW@TU Dublin. It has been accepted for inclusion in Articles by an authorized administrator of ARROW@TU Dublin. For more information, please contact arrow.admin@tudublin.ie, aisling.coyne@tudublin.ie,gerard.connolly@tudublin.ie. Funder: NSFC (Grant No. 10672154) and SRFDP of China (Project No. 20050358010)

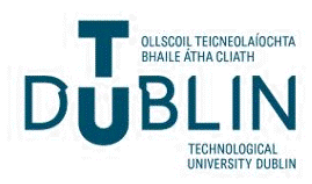




\section{Microstructures and viscoelastic properties of anisotropic magnetorheological elastomers}

\author{
L Chen ${ }^{1}$, X L Gong ${ }^{1}$ and $\mathrm{W} \mathrm{H} \mathrm{Li}^{2}$ \\ ${ }^{1}$ CAS Key Laboratory of Mechanical Behavior and Design of Materials, \\ Department of Modern Mechanics, University of Science and Technology of China, \\ Hefei 230027, People's Republic of China \\ ${ }^{2}$ School of Mechanical, Materials and Mechatronic Engineering, University of Wollongong, \\ Wollongong, NSW 2522, Australia \\ E-mail: gongxl@ustc.edu.cn
}

Received 7 June 2007, in final form 10 October 2007

Published

Online at stacks.iop.org/SMS/16/1

\begin{abstract}
The microstructures and viscoelastic properties of anisotropic magnetorheological elastomers are investigated. The measurement results show that their mechanical properties are greatly dependent on the magnetic flux density applied during preparation. A finite-column model is proposed to describe the relationships between the microstructures and the viscoelastic properties. The simulation results agree well with the experimental results.
\end{abstract}

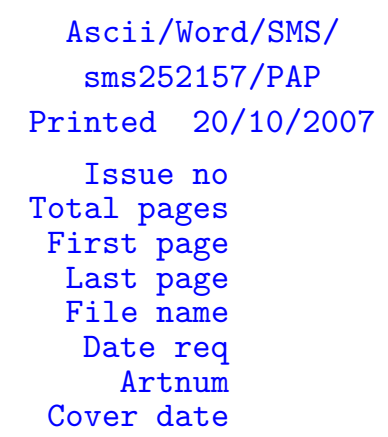

Q.1 (Some figures in this article are in colour only in the electronic version)

\section{Introduction}

Magnetorheological elastomers (MREs) are a class of smart materials whose mechanical properties (such as shear modulus) can be controlled by an external magnetic field [1-5]. This is achieved via adding the micro-sized magnetizable particles into a matrix. By curing the mixture in the presence of a magnetic field, field-induced interactions between particles promote them to form anisotropic ordered pre-configurations such as chains or columns aligned along the field direction. These structures help to improve the field-dependence of MREs' mechanical properties when the MREs are exposed to an applied magnetic field. Because of these special properties, MREs have attracted increasing attention and have recently gained broad application prospects. The field-dependent mechanical properties of the MREs have been investigated experimentally [6-8]. Several MRE based devices have been reported [3, 9].

Some theoretical models have also been developed to describe the behavior of MREs. Jolly et al [10] proposed a dipole model based on the magnetic interactions between two adjacent particles. Davis [11] calculated the field-induced shear modulus by assuming that the particles form an infinite chain in the matrix. Zhu et al [12] considered the interaction between particle chains in MREs. These models have played important roles in practical applications. However, most of these researches have assumed that perfect infinite chain structures are formed in MREs, which is not in good agreement with experimental results.

This paper is aimed at finding the determining factor in the formation of ordered microstructures in anisotropic MREs and the relation between their microstructures and viscoelastic properties. A finite-column model is proposed to explain the experimental results.

\section{Experimental details}

\subsection{Preparation of MRE materials}

A group of MREs are prepared by curing a mixture of carbonyl iron particles, natural rubber, and other additives under external fields with magnetic flux densities of $B_{\text {pre }}=0,200,400,600$, 800 , and $1000 \mathrm{mT}$ respectively. In order to observe the MREs' microstructures clearly, a low volume fraction of iron particles is used. To this end, the volume fractions of iron particles for all samples are $11 \%$. The carbonyl iron particles are provided by BASF (German) and have an average diameter of $3.5 \mu \mathrm{m}$. The natural rubber, plasticizer, and other additives are provided by Hefei Wangyou Rubber Company of China. 


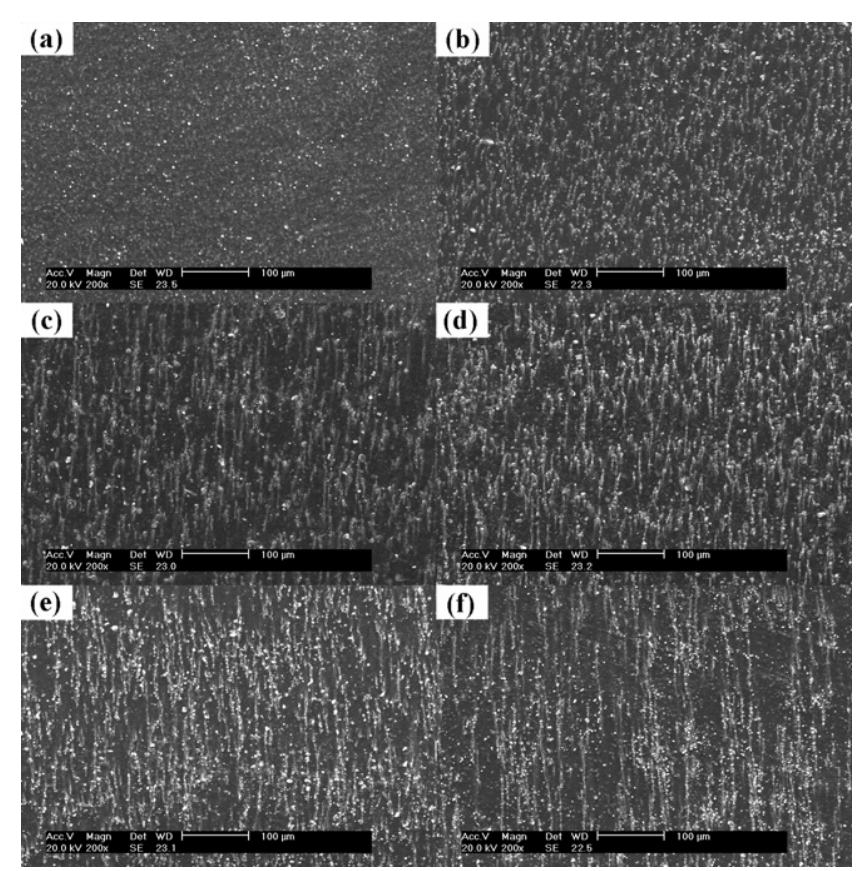

Figure 1. SEM images with 200 times magnification of MREs prepared under magnetic flux density $B_{\text {pre }}$ of (a) $0 \mathrm{mT}$, (b) $200 \mathrm{mT}$, (c) $400 \mathrm{mT}$, (d) $600 \mathrm{mT}$, (e) $800 \mathrm{mT}$, (f) $1000 \mathrm{mT}$.

The fabrication of MREs consists of three major steps: mixing, forming pre-configuration and sulfuration. The mixture is processed with conventional rubber-mixing techniques. A Double-Roll Mill (Taihu Rubber Machinery. Inc. China, Model XK-160) is used to mix the crosslinkers, the processing aids, the carbonyl iron particles, and the plasticizer with the natural rubber homogeneously. The mixture is then compression-molded into a mold in a self-modified magnet heat coupled device for pre-configuration and sulfuration. During the pre-configuration stage, the heating system and the magnetic field are both turned on. The particles are magnetized and then form anisotropic structures. $30 \mathrm{~min}$ later, shutting down the magnetic field, the temperature is raised to $153^{\circ} \mathrm{C}$. Under these conditions, the sample is sulfurated for $15 \mathrm{~min}$. Then the MRE sample is prepared.

\subsection{Observation of microstructure}

To observe the microstructure, samples are cut into pieces (with a surface area of $3 \mathrm{~mm} \times 3 \mathrm{~mm}$ ). Each piece's surface is coated with a thin layer of gold and then placed into an environmental scanning electronic microscope (SEM, Philip of Holland, Model of XT30 ESEM-MP). The microstructures of samples are observed at an accelerating voltage of $20 \mathrm{kV}$.

\subsection{Measurement of MREs' viscoelastic property}

A dynamic mechanical analyser (DMA) is the common equipment for dynamic testing on viscoelastic materials. To measure the MREs' viscoelastic properties under an external magnetic field, the DMA (Triton Technology Ltd UK, Model Tritec 2000B) is modified by introducing a self-made electromagnet which can generate a variable magnetic flux density up to $1 \mathrm{~T}$. This system applies a fixed oscillatory strain to the specimen and measures the amplitude and phase of the

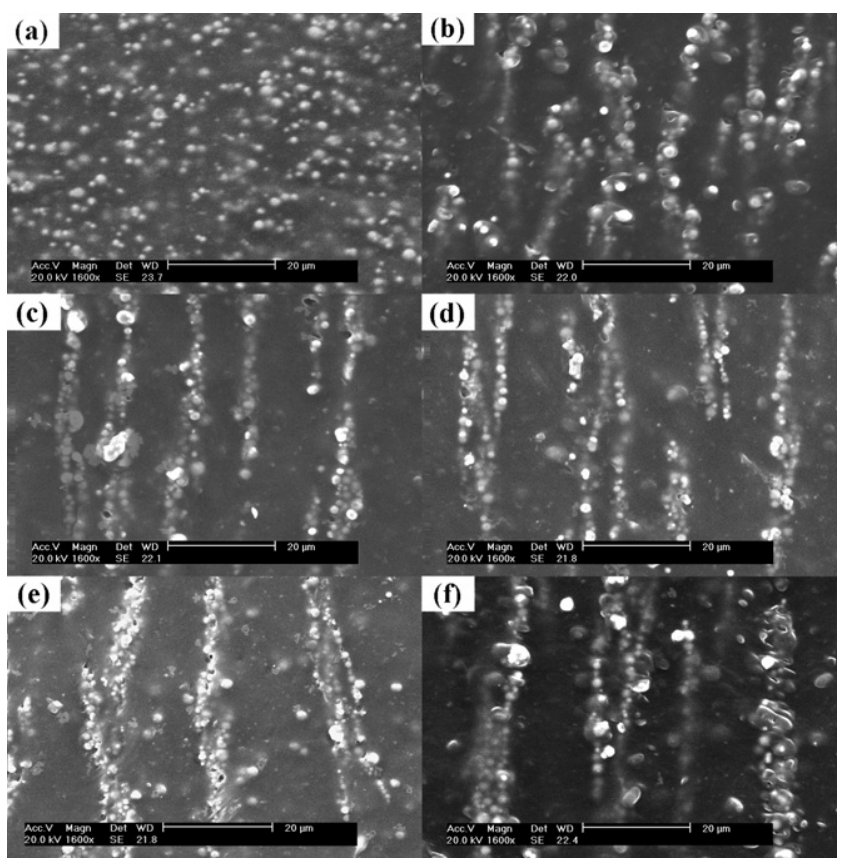

Figure 2. SEM images with 1600 times magnification of MREs prepared under magnetic flux density $B_{\text {pre }}$ of (a) $0 \mathrm{mT}$, (b) $200 \mathrm{mT}$, (c) $400 \mathrm{mT}$, (d) $600 \mathrm{mT}$, (e) $800 \mathrm{mT}$, (f) $1000 \mathrm{mT}$.

output force. It can measure the MREs' shear modulus and loss factor at different magnetic fields, different frequencies, and different dynamic stain amplitudes respectively.

In the experiments, the range of the external magnetic field is $0-600 \mathrm{mT}$, the driving frequency is fixed as $1 \mathrm{~Hz}$, and the dynamic strain amplitude is set as $0.3 \%$. The experiments are carried out at room temperature.

\section{Results}

\subsection{Microstructure}

Some MREs are prepared under different magnetic flux densities of $B_{\text {pre }}=0,200,400,600,800$, and $1000 \mathrm{mT}$ respectively. Figures 1 and 2 show the SEM images of these MREs. Figures 1 and 2 have magnifications of 200 and 1600 times respectively. The white spots are the iron particles and the black background is the matrix. In images (a), the particles show random distribution. The corresponding MRE is prepared without magnetic field, so there is no magnetic interaction between particles in preparation. The effect of Brownian motion makes the particles arrange themselves in a random distribution. In images (b), the particles form short and thin columns, and the space between the columns is small. From images (b)-(f), with an increment of $B_{\text {pre }}$, the thickness of the columns and the space between them are increased. It can be found that the configuration of particles is determined by the magnetic flux density applied during the preparation. During preparation, when the magnetic field is applied, the particles are magnetized and attracted to each other. They begin to move and form the column structures after the magnetic force overcomes the matrix resistance. With the increase of $B_{\text {pre }}$, the interaction between the particles is increased. More particles aggregate with each other, so the 


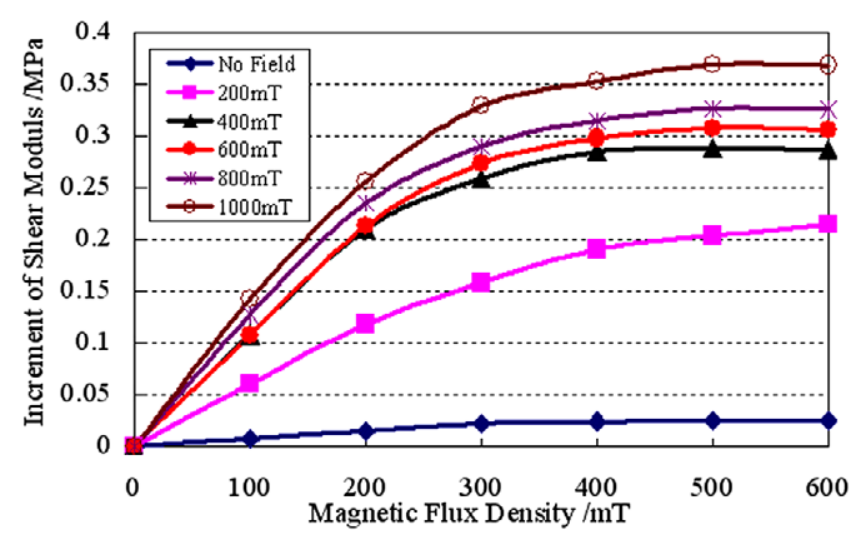

Figure 3. Increment of the field-induced shear modulus of MREs prepared under different magnetic flux densities.

columns become longer and thicker. This leads to an increase in the volume of the columns, so the space between the columns is increased accordingly.

\subsection{Viscoelastic properties}

When MREs are exposed to an external magnetic field, their shear modulus can be divided into two parts: one is the natural shear modulus $\left(G_{0}\right.$, initial modulus without field) and the other is the field-induced modulus ( $\Delta G$, caused by the external magnetic field). Here, the natural shear modulus of each sample is about 1.3 MPa. There is little influence of the configuration of particles on the natural shear modulus because the volume fraction of iron particles is on a low level. Figure 3 shows the field-induced shear modulus of the MREs prepared in magnetic flux densities $B_{\text {pre }}$ of $0,200,400,600$, $800,1000 \mathrm{mT}$. The field-induced shear modulus of each sample shows an increasing trend with increased magnetic flux density applied. This is because the increment of shear modulus comes from the attraction of magnetized particles. The stronger the applied magnetic field is, the stronger the attraction is, and the harder the MRE is. After certain magnetic fields, the particles are nearly saturated, and the actions between magnetizable particles cannot vary with the applied magnetic flux density. The field-induced modulus thus reaches the maximum $\left(\Delta G_{\mathrm{s}}\right.$, the saturated field-induced modulus). It can also be seen from figure 3 that the modulus of the $200 \mathrm{mT}$ sample is still increasing under the high field. It implies that the high field has some influence on the degree of order of particles in that sample. The particles tend to form a more ordered configuration which leads to an increase of the saturated fieldinduced modulus of the $200 \mathrm{mT}$ sample. By comparing results of these six samples, it is also found that the sample prepared under a strong magnetic flux density $B_{\text {pre }}$, has a high fieldinduced modulus. For example, the saturated field-induced modulus of MRE prepared in $B_{\text {pre }}$ of $1000 \mathrm{mT}$ is $0.36 \mathrm{MPa}$ while another prepared at a zero-field is only $0.03 \mathrm{MPa}$.

\section{A finite-column model}

It is shown in figures 1 and 2 that for a certain MRE, the particle columns have a finite length and the same thickness, and the spaces between adjacent columns are also nearly the
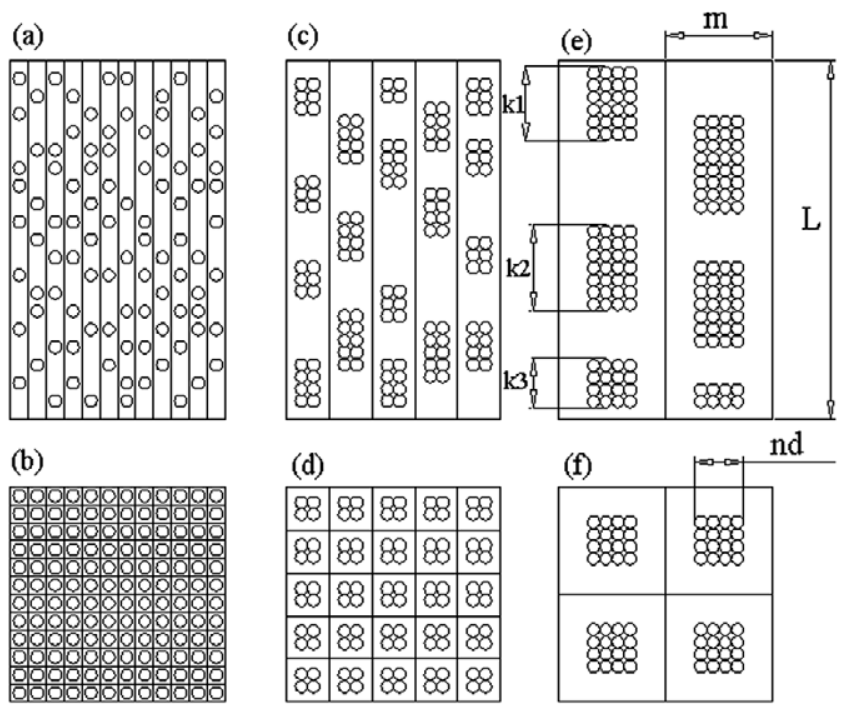

Figure 4. A sketch of MREs' finite-column model. MREs are prepared under (a), (b) zero field, (c), (d) low field and (e), (f) strong field respectively. (a), (c), and (e) are viewed in a direction parallel to the particle column, and (b), (d), and (f) are viewed in a direction perpendicular to the particle column.

same. A physical model, shown in figure 4, is proposed to describe these experimental results. It sketches the structures of MREs prepared under zero field, low field, and strong field, respectively. Images (a), (c), and (e) are viewed in a direction parallel to the particle column, and (b), (d), and (f) are in a direction perpendicular to the particle column. The circles represent the particles and the white background represents the matrix. In these images, the columns' thickness ( $n d, n$ is the number of the aggregated particles, $d$ is the particles' diameter), the columns' total length $\left(\sum k_{i}\right)$, and the spaces between the columns $(\mathrm{m})$ are all increased with the magnetic field applied during preparation. Based on figures 1 and 2, the following three points are assumed. Firstly, the MRE can be divided into several blocks with the same volume (by the dimensions of $m \times m \times L)$. Secondly, several quadrate columns with the same thickness ( $n d)$ and random length are contained in each block. Thirdly, particles in all the blocks have the same volume per cent.

From the above presumption, it is known that particles in each block have the same volume per cent as that in the whole $\operatorname{MRE}(\varphi)$. So in each block, the relationship between these parameters can be written as:

$$
\sum k_{i} / L=6 \varphi /\left[\pi(n d / m)^{2}\right]
$$

where the numerical values of $m / n d$ can be obtained from the SEM images (shown in figures 1 and 2). But in each SEM image, the thickness of the columns and the spaces between them are not uniform. So the law of averages is used. We draw three lines (not shown in the images) to divide each image in figures 1 and 2 into four equal parts. These lines thread the matrix and are perpendicular to the column axes. By adding the length of particle-columns across the three lines in the corresponding images in figures 1 and 2, the value of $\sum n d$ is obtained. Using the same method, by adding the length of the matrix across the three lines, the value of $\sum m$ is obtained. Then the average value of $n d / m$ is accounted for 
Table 1. The average values of $n d / m$ and $\sum k_{i} / L$ of MREs prepared in different magnetic fields.

\begin{tabular}{lllllll}
\hline & \multicolumn{6}{c}{$B_{\text {pre }}(\mathrm{mT})$} \\
\cline { 2 - 7 } & 0 & 200 & 400 & 600 & 800 & 1000 \\
\hline$n d / m$ & 0.84 & 0.68 & 0.62 & 0.59 & 0.56 & 0.55 \\
$\Sigma k_{i} / L$ & 0.29 & 0.45 & 0.55 & 0.60 & 0.67 & 0.71 \\
\hline
\end{tabular}

as $\sum n d / \sum m$. The average values of $n d / m$ and $\sum k_{i} / L$ of MREs prepared in different magnetic fields are list in table 1.

The results of $\sum k_{i} / L$ show a rising trend with $B_{\text {pre }}$ which agreed well with that indicated in figures 1 and 2 . If the length of the columns is infinite, $\sum k_{i} / L$ will approach 1 . In this case, a very strong magnetic field must be applied during the preparation.

When a shear strain is applied on the electrorheological fluids (ERF), the ERF's dielectric is changed which leads to an electrical field-induced shear stress. The electrical fieldinduced shear stress can be expressed as [13]

$$
\tau=-\frac{1}{2} \varepsilon_{0} \frac{\partial K_{\mathrm{eff}}}{\partial \theta} E_{0}^{2}
$$

where $E_{0}$ is the applied electrical field strength, $K_{\text {eff }}$ is the effective dielectric constant of the ERF in the direction of the applied field. According to the electromagnetism [14], the electricity and magnetism always have the corresponding physical law. So a similar phenomenon will be happened in the MRE. The MRE's magnetic susceptibility is changed which leads to a magnetic field-induced shear stress. the corresponding magnetic field-induced shear stress can be expressed as

$$
\tau=-\frac{1}{2} \mu_{0} \frac{\partial \chi_{z z}}{\partial \theta} H_{0}^{2}
$$

where

$$
\chi_{z z}=\chi_{\|} \cos ^{2} \theta+\chi_{\perp} \sin ^{2} \theta
$$

and $H_{0}$ is the applied magnetic field strength, $\chi_{z z}$ is the MRE's effective magnetic susceptibility in the direction of the applied field, $\theta$ is the shear strain. $\chi_{z z}$ can be analysed into $\chi_{\|}$ and $\chi_{\perp}$, which are parallel and perpendicular to the particle column respectively. The effective magnetic permeability of the composite can be predicted by Wiener bounds $[15,16]$; if the elements of composites are in a series arrangement, then the effective magnetic permeability will reach a minimum, but when the elements of the composites are in a parallel connection, the effective magnetic permeability will reach a maximum. They can be expressed as:

$$
\begin{gathered}
\mu_{\mathrm{eff}, \max }=\phi \mu_{\alpha}+(1-\phi) \mu_{\beta} \\
\mu_{\mathrm{eff}, \min }=\mu_{\alpha} \mu_{\beta} /\left[\phi \mu_{\beta}+(1-\phi) \mu_{\beta}\right]
\end{gathered}
$$

where $\mu_{\alpha}$ and $\mu_{\beta}$ are the relative permeabilities of elements of $\alpha$ and $\beta$ respectively, and $\phi$ is the volume fraction of element $\alpha$. In our model, each block has the same magnetic property as the whole MRE sample. So the permeability of one block (shown in figure 5(a)) is investigated next.

The block can be regarded as a combination of some particle-units and a matrix which is assembled in a series arrangement and parallel connection repeated several times. (a)

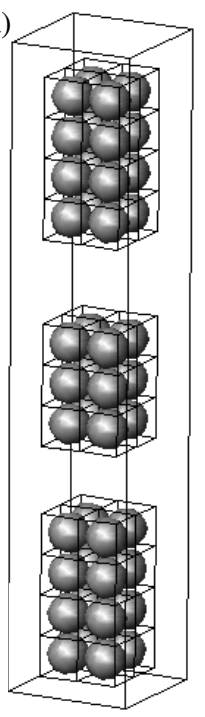

(b)

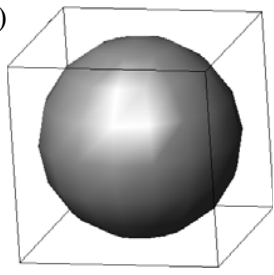

(c)

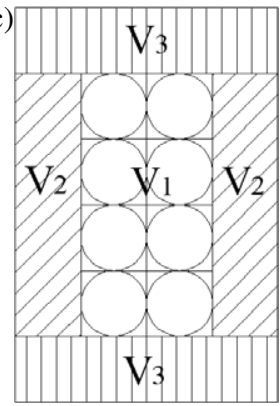

Figure 5. Sketch of the (a) block, (b) basic particle-unit, and (c) section parallel to the axes of the particle column in the block.

The basic particle-unit (shown in figure 5(b)) is a cube inscribed by a particle. This inscribed figure is not in an ordered arrangement (such as series and parallel), but is an isotropic structure. So its effective magnetic permeability $\mu_{p u}$ is not suitable to be calculated by the Wiener bound as in equations (5) and (6), and a more general Maxwell Garnett mixing rule [17] is used:

$$
\mu_{p u}=\mu_{\mathrm{m}}+2 f \mu_{\mathrm{m}}\left(\mu_{\mathrm{p}}-\mu_{\mathrm{m}}\right) /\left[\mu_{\mathrm{p}}+\mu_{\mathrm{m}}-f\left(\mu_{\mathrm{p}}-\mu_{\mathrm{m}}\right)\right]
$$

where $\mu_{\mathrm{m}}$ and $\mu_{\mathrm{p}}$ are the effective magnetic permeability of the matrix and particles respectively, and $f$ is a particle's volume percentage in a cube particle-unit. In this particle-unit, we have $f=(4 / 3) \pi R^{3} /(2 R)^{3}=\pi / 6$. Figure 5(c) shows the section parallel to the axes of the particle column in the block. Region $V_{1}$ is the particle columns, and regions $V_{2}$ and $V_{3}$ are the surrounding matrix. So we can obtain the effective magnetic permeability of the block by the Wiener bound. In the direction parallel to the particle column, regions $V_{1}$ and $V_{2}$ are on the parallel connection, and the permeability can be expressed by:

$$
\mu_{12}=\mu_{v_{1}+v_{2}}=(n d / m)^{2} \mu_{p u}+\left[1-(n d / m)^{2}\right] \mu_{\mathrm{m}} .
$$

Regions $V_{12}$ and $V_{3}$ are in a series arrangement, so the permeability of the block parallel to the column axes can be expressed by:

$$
\mu_{\|}=\mu_{12} \mu_{\mathrm{m}} /\left[\left(\sum k_{i} / L\right) \mu_{\mathrm{m}}+\left(1-\sum k_{i} / L\right) \mu_{12}\right] .
$$

Using the same methods, the permeability of the block perpendicular to the column axes can be expressed by:

$$
\mu_{\perp}=\left(\sum k_{i} / L\right) \mu_{a}+\left(1-\sum k_{i} / L\right) \mu_{\mathrm{m}}
$$

where

$$
\mu_{a}=(n d / m) \mu_{b}+(1-n d / m) \mu_{\mathrm{m}}
$$

where

$$
\mu_{b}=\mu_{p u} \mu_{\mathrm{m}} /\left[(n d / m) \mu_{\mathrm{m}}+(1-n d / m) \mu_{p u}\right] .
$$




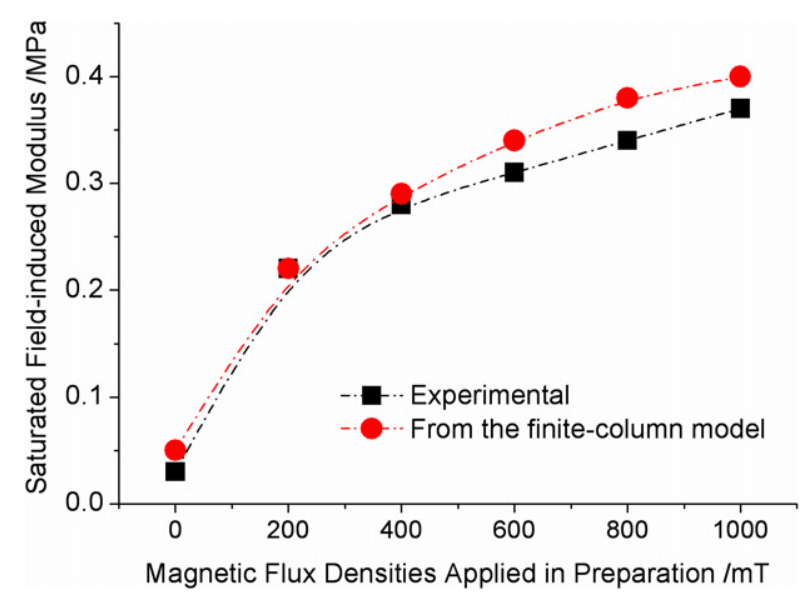

Figure 6. Saturated field-induced shear modulus of MREs prepared under different magnetic flux densities.

From equations (1) to (11), the field-induced shear modulus can be calculated as:

$$
G=\tau / \theta=\left(\mu_{\|}-\mu_{\perp}\right) H_{0}^{2} \sin \theta \cos \theta / \theta .
$$

\section{Discussions}

In the finite-column model, there are three undetermined parameters of $m / n d, H_{0}$, and $\theta$ respectively. Therefore, the model can calculate the field-induced shear modulus of MREs prepared in different fields and performed on different external fields and strains.

In our experiment, the average values of $n d / m$ of MREs prepared in different magnetic fields are list in table $1 . \theta$ is set at 0.003 in the dynamic testing. When the fieldinduced modulus reaches a maximum $\left(\Delta G_{\mathrm{s}}\right)$, the external magnetic flux density $B_{0}$ is $600 \mathrm{mT}$. The corresponding external magnetic field strength $H_{0}$ is $0.48 \mathrm{MA} \mathrm{m}^{-1}$. Then $\mu_{\mathrm{p}}$ and $\mu_{\mathrm{m}}$ are about 1000 and 1 respectively. The saturated fieldinduced shear modulus is calculated by introducing the above parameters. The calculated results are shown and compared to the experimental results in figure 6 . We can see that the two results are consistent with each other. The finite-column model seems suitable to calculate the field-induced shear modulus of the MREs with different microstructures.

Some theoretical models have been developed to describe the behavior of MREs. Jolly et al [10] set up a dipole model based on the magnetic interactions between two adjacent particles. The saturated field-induced shear modulus is deduced as:

$$
G_{J}=\varphi J_{\mathrm{s}}^{2} / 2 \mu_{\mathrm{p}} \mu_{0} h^{3} .
$$

Davis [11] assumed that the particles form an infinite chain in the matrix. The saturated field-induced shear modulus is calculated by

$$
G_{D}=3 \varphi \mu_{0} M_{\mathrm{s}}^{2} d^{3} / 5 h^{3} .
$$

In the two models above, $h$ is the space between two adjacent particles in a chain. If $h$ reaches the minimal value, it will be equal to the diameter of the particle $(d)$. Then the shear modulus will reach the maximum. Inducing the value of constants $\varphi$ and $d$ in this paper into equations (14) and (15), we can find that $G_{J}=0.11 \mathrm{MPa}$ and $G_{D}=0.19 \mathrm{MPa}$. Compared to the experimental data in figure 6 , the results of these two models are close to the data of MREs prepared in low fields. In fact, the particles in MREs prepared in low fields form a chain structure, while these models are both based on the chain structure assumption. So these models are not suitable for MREs prepared in high fields or with the column structure. However, in order to get the high MR effect, the MREs are often prepared in high fields. In this case, the finite-column model is important for predicting the performance of MREs because of its wide applicability.

In the semi-empirical equations developed by Jolly et al and Davis, they both contain an undetermined parameter $h$. Actually, the particles in MREs hardly form the head-to-tail structure. Therefore, the value of $h$ is difficult to confirm even when the SEM images are obtained. In the finite-column model, the undetermined parameter $n d / m$ is easily confirmed by the law of averages. This method can make the model approach a satisfactory precision.

\section{Conclusions}

A group of MREs are prepared in different magnetic fields. Their microstructures are observed by an environmental scanning electronic microscope (SEM). The results show that the microstructures are greatly affected by the magnetic flux density during the preparation $\left(B_{\text {pre }}\right)$. With the increment of $B_{\text {pre }}$, the length and thickness of the particle columns are increased. The MREs' viscoelastic properties are also tested by a mechanical-magnetic coupling dynamic mechanical analyser (DMA). The results show that the field-dependence of MREs' viscoelastic properties increase with the applied magnetic flux densities during testing. The increment is also determined by $B_{\text {pre. }}$ MREs prepared under high magnetic fields have a large field-induced shear modulus and high MR effect.

A finite-column model is proposed to calculate the fieldinduced shear modulus from the observation of microstructures. The semi-empirical simulation shows that it agrees well with the experimental results. It is suitable for MREs which are prepared in different magnetic fields and with different microstructures.

\section{Acknowledgments}

Financial support from NSFC (Grant No. 10672154) and SRFDP of China (Project No. 20050358010) is gratefully acknowledged. The Scholarship BRJH funding of the Chinese Academy of Sciences is also appreciated.

\section{References}

[1] Carlson J D and Jolly M R 2000 MR fluid, foam and elastomer devices Mechatronics 10 555-69

[2] Jolly M R, Carlson J D, Munoz B C and Bullions T A 1996 The magnetoviscoelastic response of elastomer composites consisting of ferrous particles embedded in a polymer matrix J. Intell. Mater. Syst. Struct. 7 613-22

[3] Ginder J M, Nichols M E, Elie L D and Clark S M 2000 Controllable-stiffness components based on magnetorheological elastomers Proc. SPIE 3985 418-25

[4] Zhou G Y and Li J R 2003 Dynamic behavior of a magnetorheological elastomer under uniaxial deformation: I. Experiment Smart Mater. Struct. 12 859-72 
[5] Lokander M, Reitberger T and Stenberg B 2004 Oxidation of natural rubber-based magnetorheological elastomers Polym. Degrad. Stabil. 86 467-71

[6] Bellan C and Bossis G 2002 Field dependence of viscoelastic properties of MR elastomers Int. J. Mod. Phys. B $162447-53$

[7] Zhou G Y 2003 Shear properties of a magnetorheological elastomer Smart Mater. Struct. 12 139-46

[8] Chen L, Gong X L, Jiang W Q, Yao J J, Deng H X and Li W H 2007 Investigation on magnetorheological elastomers based on natural rubber J. Mater. Sci. 42 5483-9

[9] Deng H X, Gong X L and Wang L H 2006 Development of an adaptive tuned vibration absorber with magnetorheological elastomer Smart Mater. Struct. 15 N111-6

[10] Jolly M R, Carlson J D and Munoz B C 1996 A model of the behaviour of magnetorheological materials Smart Mater. Struct. 5 607-14
[11] Davis L C 1999 Model of magnetorheological elastomers J. Appl. Phys. 85 3348-51

[12] Zhu Y S, Gong X L, Dang H, Zhang X Z and Zhang P Q 2006 Numerical analysis on magnetic-induced shear modulus of magnetorheological elastomers based on multi-chain model Chin. J. Chem. Phys. 19 126-30

[13] Davis L C 1992 Polarization forces and conductivity effects in ER fluids J. Appl. Phys. 72 1334-40

[14] Hallen E 1962 Electromagnetic Theory (London: Chapman and Hal1) p 122

[15] Wiener O 1912 Die theorie des mischkorpers fur das feld der stationaren stromung Kl. Königl. Saechs. Ges. 32 509-14

[16] Karkkainen K K, Sihvola A H and Nikoskinen K I 2000 Effective permittivity of mixtures: numerical validation by the FDTD method IEEE Trans. Geosci. Remote 38 1303-8

[17] Maxwell-Garnett J C 1904 Colour in metal glasses and metallic films Phil. Trans. R. Soc. 203 385-91 


\section{Queries for IOP paper 252157}

Journal: $\quad$ SMS

Author: $\quad$ L Chen et al

Short title: Microstructures and viscoelastic properties of anisotropic magnetorheological elastomers

\section{Page 1}

Query 1:

Author: Please be aware that the colour figures in this proof will normally only appear in colour in the online Web version. If you require colour in the printed journal and have not previously arranged it, please contact the Publishing Administrator now.

\section{Page 4}

Query 2:

Author: This sentence has been reworded. Please check.

Reference linking to the original articles

References with a volume and page number in blue have a clickable link to the original article created from data deposited by its publisher at CrossRef. Any anomalously unlinked references should be checked for accuracy. Pale purple is used for links to e-prints at ArXiv. 